Теорія Ймовір. та Матем. Статист. Вип. 82, 2010
Theor. Probability and Math. Statist.

No. 82, 2011, Pages 129-138

S 0094-9000(2011)00832-0

Article electronically published on August 4, 2011

\title{
CONSISTENCY OF QUANTILE ESTIMATORS IN REGRESSION MODELS WITH LONG-RANGE DEPENDENT NOISE
}

UDC 519.21

\author{
I. M. SAVICH
}

\begin{abstract}
Sufficient conditions for weak consistency of the Koenker-Bassett estimator are obtained for the parameter of a nonlinear regression model with continuous time and random noise possessing the property of the long-range dependence.
\end{abstract}

\section{INTRODUCTION}

Mathematical models for observations of the form "signal plus noise" have a wide area of applications in various fields of natural and social sciences such as turbulence theory, meteorology, hydrology, geophysics, statistical radiophysics, chemical kinetics, econometrics, finance, sociology, etc.

Models with continuous time and random noise with long-range memory are of special interest. Some recent applied studies prove that various data observed in the sciences mentioned above show the property of long-range dependence (see, for example, the books by Beran [1, Leonenko [2, Doukhan et al. 3]). Parameters of nonlinear regression models can be estimated by a method proposed by Bassett and Koenker [4. The KoenkerBassett estimator can be used to estimate an unknown parameter of the unknown $\beta$ quantile of observations. The value $\beta \in(0,1)$ is defined from the distribution of the random noise.

Earlier studies [5, 6] dealt with sufficient conditions for weak consistency and asymptotic normality of the Koenker-Bassett estimator in the case of nonlinear models with discrete time and with independent and identically distributed errors of observations.

The main aim of this paper is to obtain sufficient conditions for weak consistency of the Koenker-Bassett estimator in the case of nonlinear models with continuous time.

\section{Setting of the PRoblem}

Consider the following nonlinear regression model:

$$
X(t)=g(t, \theta)+\varepsilon(t), \quad t \geq 0,
$$

where $g(t, \theta)$ is a real function that is continuous with respect to the set of all its arguments $(t, \theta) \in \mathbb{R}_{+} \times \Theta^{c}, \Theta \subset \mathbb{R}^{q}$ is an open bounded set of parameters containing $\theta$, and $\Theta^{c}$ is the closure in $\mathbb{R}^{q}$ of the set $\Theta$.

We also assume that $\varepsilon(t)$ satisfies the following conditions.

2010 Mathematics Subject Classification. Primary 62J02; Secondary 62J99.

Key words and phrases. Quantile estimators, consistency, nonlinear regression models, noise with long-range memory. 
A1. $\varepsilon(t), t \in \mathbb{R}$, is a local functional of the Gaussian stationary process $\xi(t)$, that is, $\varepsilon(t)=G(\xi(t))$, where $G(x), x \in \mathbb{R}$, is a Borel function; moreover,

$$
\mathrm{E} \varepsilon(0)=0, \quad \mathrm{E} \varepsilon^{2}(0)<\infty .
$$

A2. $\xi(t), t \in \mathbb{R}$, is a measurable real stationary Gaussian process defined on the probability space $(\Omega, \mathfrak{F}, \mathrm{P})$. Furthermore, $\xi(t)$ is a mean square continuous process with long-range dependence, and its covariance function is given by

$$
\mathrm{E} \xi(t) \xi(0)=B(t)=L(|t|) /|t|^{\alpha}, \quad \alpha \in(0,1),
$$

where $L(t)$ is a slowly varying at infinity function (see, for example, 7]). Finally we assume that

$$
\mathrm{E} \xi(t)=0, \quad \mathrm{E} \xi^{2}(t)=B(0)=1 .
$$

We denote by $F(x)$ the distribution function of $\varepsilon(0)$ and assume that

A3. $F(0)=\beta$ for some $\beta \in(0,1)$.

Consider the function

$$
\rho_{\beta}(x)=\left\{\begin{array}{ll}
\beta x, & x \geq 0, \\
(\beta-1) x, & x<0,
\end{array} \quad \beta \in(0,1) .\right.
$$

Definition 1. Any random vector $\hat{\theta}_{T}=\hat{\theta}_{T}(X(t), t \in[0, T]) \in \Theta^{c}$ such that

$$
Q_{T}\left(\hat{\theta}_{T}\right)=\inf _{\tau \in \Theta^{c}} Q_{T}(\tau)
$$

where

$$
Q_{T}(\tau)=\int_{0}^{T} \rho_{\beta}(X(t)-g(t, \tau)) d t,
$$

is called the Koenker-Bassett estimator (or generalized least modules estimator) of the unknown parameter $\theta \in \Theta$ constructed from observations $X(t), t \in[0, T]$, represented in the form of (10) with respect to the loss function $\rho_{\beta}(x), x \in \mathbb{R}$.

The estimator $\hat{\theta}_{T}$ exists if the above conditions are satisfied for the model (1) (see, for example, 8] ).

Since

$$
\mathrm{P}(X(t)<g(t, \theta))=\mathrm{P}(\varepsilon(t)<0)=\mathrm{P}(\varepsilon(0)<0)=\beta,
$$

the model of observations (11) can be viewed as a nonlinear quantile regression. Indeed, $\hat{\theta}_{T}$ is an estimator of the unknown parameter $\theta$ for the $\beta$-quantiles $g(t, \theta)$ of the observations $X(t), t \in[0, T]$.

Let $g(t, \tau)$ be a continuously differentiable function with respect to $\tau \in \Theta$ and let

$$
\begin{gathered}
g_{i}(t, \tau)=\frac{\partial}{\partial \tau_{i}} g(t, \tau), \\
d_{T}^{2}(\theta)=\operatorname{diag}\left(d_{i T}^{2}(\theta)\right)_{i=1}^{q}, \quad d_{i T}^{2}(\theta)=\int_{0}^{T} g_{i}^{2}(t, \theta) d t, \\
\liminf _{T \rightarrow \infty} T^{-1 / 2} d_{i T}(\theta)>0, \quad i=1, \ldots, q .
\end{gathered}
$$

The latter lower limit can even be equal to infinity.

Now we change the variables in the regression function as follows: we let $u=$ $T^{-1 / 2} d_{T}(\theta)(\tau-\theta)$ and put

$$
h(t, u)=g\left(t, \theta+T^{1 / 2} d_{T}^{-1}(\theta) u\right),
$$

where $\theta$ is the true value of the parameter. The image of the parametric set $\Theta$ under this transformation is $\tilde{U}_{T}(\theta)=T^{-1 / 2} U_{T}(\theta)$, where $U_{T}(\theta)=d_{T}(\theta)(\Theta-\theta)$. The idea behind 
this change of variables is that the Koenker-Bassett estimator $\hat{\theta}_{T}$ is transformed into the normalized vector $\bar{u}_{T}=T^{-1 / 2} d_{T}(\theta)\left(\hat{\theta}_{T}-\theta\right)$.

Denote $Q_{T}^{*}(u)=Q_{T}\left(\theta+T^{1 / 2} d_{T}^{-1}(\theta) u\right), u \in \tilde{U}_{T}^{c}(\theta)$,

$$
\begin{gathered}
\Phi_{k T}\left(u_{1}, u_{2}\right)=\int_{0}^{T}\left|h\left(t, u_{1}\right)-h\left(t, u_{2}\right)\right|^{k} d t, \quad k=1,2, \\
\Psi_{T}\left(u_{1}, u_{2}\right)=\int_{0}^{T} \rho_{\beta}\left(h\left(t, u_{1}\right)-h\left(t, u_{2}\right)\right) d t, \quad u_{1}, u_{2} \in \tilde{U}_{T}^{c}(\theta), \\
\varepsilon^{+}(t)=\max (\varepsilon(t), 0) .
\end{gathered}
$$

\section{Some Properties of the LOSS FUNCTION}

Let $\beta=\min \{\beta, 1-\beta\}$ and $\bar{\beta}=\max \{\beta, 1-\beta\}$. We list below some useful properties of the loss function $\rho_{\beta}$ (the proofs of these properties are straightforward).

I. $\rho_{\beta}(a x)=a \rho_{\beta}(x), a \geq 0$.

II. $\rho_{\beta}(x)+\rho_{\beta}(-x)=|x|$.

III. $\beta|x| \leq \rho_{\beta}(x) \leq \bar{\beta}|x|$.

IV. $\bar{\rho}_{\beta}(x+y) \leq \rho_{\beta}(x)+\rho_{\beta}(y)$.

V. $\left|\rho_{\beta}(x)-\rho_{\beta}(y)\right| \leq \max \left\{\rho_{\beta}(x-y), \rho_{\beta}(y-x)\right\} \leq \bar{\beta}|x-y|$.

VI. If the first moment of a random variable $\xi$ exists, then

$$
\mathrm{E} \rho_{\beta}(\xi)=\mathrm{E} \rho_{1-\beta}(-\xi) .
$$

VII. If the second moment of a random variable $\xi$ exists, then

$$
\operatorname{Var} \rho_{\beta}(\xi)=\operatorname{Var} \rho_{1-\beta}(-\xi) .
$$

It follows from property IV that

$$
\rho_{\beta}(x-y) \geq \rho_{\beta}(x)-\rho_{\beta}(y) .
$$

If we additionally assume that $\mathrm{E} \xi=0$, then

$$
\mathrm{E} \rho_{\beta}(\xi)=\mathrm{E} \rho_{\beta}(-\xi)=\mathrm{E} \rho_{1-\beta}(\xi)=\mathrm{E} \rho_{1-\beta}(-\xi)=\mathrm{E} \xi^{+} .
$$

Properties VI and $\mathrm{E} \varepsilon(t)=0, t \in \mathbb{R}$, imply that

$$
\mathrm{E} \rho_{\beta}(\varepsilon(t))=\mathrm{E} \rho_{\beta}(\varepsilon(0))=\mathrm{E} \varepsilon^{+}(0) .
$$

\section{Main RESUlt}

In what follows we need the following conditions.

B. (i) For all $\varepsilon>0$ and $r>0$, there exists $\delta=\delta(r, \varepsilon)>0$ such that

$$
\sup _{\substack{u_{1}, u_{2} \in V^{c}(r) \cap \tilde{U}_{T}^{c}(\theta),\left\|u_{1}-u_{2}\right\| \leq \delta}} T^{-1} \Phi_{1 T}\left(u_{1}, u_{2}\right) \leq \varepsilon,
$$

where $V^{c}(r)=\left\{u \in \mathbb{R}^{q}:\|u\| \leq r\right\}$.

(ii) For all $r>0$, there exists a constant $\sigma=\sigma(r)<\infty$ such that

$$
\sup _{u \in V^{c}(r) \cap \tilde{U}_{T}^{c}(\theta)} T^{-1} \Phi_{2 T}(u, 0) \leq \sigma .
$$

C. For all $r>0$, there exists $\Delta(r)>0$ such that

$$
\inf _{u \in \tilde{U}_{T}^{c}(\theta) \backslash V^{c}(r)} T^{-1} \mathrm{E} Q_{T}^{*}(u) \geq \mathrm{E} \varepsilon^{+}(0)+\Delta(r)
$$


for sufficiently large $T>T_{0}$, and moreover

$$
\Delta\left(r_{0}\right)=a_{0} \mathrm{E} \varepsilon^{+}(0)+\Delta_{0}
$$

for some $r_{0}>0$, where $a_{0}>2$ and $\Delta_{0}>0$ are some numbers.

Theorem 1. Let $\varepsilon(t)$ satisfy conditions $\mathbf{A} 1, \mathbf{A 2}$, and $\mathbf{A} 3$. If conditions $\mathbf{B}$ and $\mathbf{C}$ hold, then

for all $r>0$.

$$
\mathrm{P}\left(\left\|\bar{u}_{T}\right\| \geq r\right)=O(B(T)) \quad \text { as } T \rightarrow \infty
$$

Proof. Put $\delta_{T}(\theta, u)=Q_{T}^{*}(u)-\mathrm{E} Q_{T}^{*}(u)$,

$$
\begin{aligned}
\delta_{T}(\theta, 0) & =Q_{T}^{*}(0)-\mathrm{E} Q_{T}^{*}(0)=Q_{T}(\theta)-\mathrm{E} Q_{T}(\theta)=\int_{0}^{T} \rho_{\beta}(\varepsilon(t)) d t-\int_{0}^{T} \mathrm{E} \rho_{\beta}(\varepsilon(t)) d t \\
& =\int_{0}^{T} \rho_{\beta}(\varepsilon(t)) d t-T \mathrm{E} \varepsilon^{+}(0) .
\end{aligned}
$$

According to the definition of the Koenker-Bassett estimator,

$$
\begin{gathered}
Q_{T}^{*}\left(\bar{u}_{T}\right)=\inf _{u \in \tilde{U}_{T}^{c}(\theta)} Q_{T}^{*}(u), \quad Q_{T}^{*}(u)=\int_{0}^{T} \rho_{\beta}(X(t)-h(t, u)) d t, \\
Q_{T}^{*}\left(\bar{u}_{T}\right) \leq Q_{T}^{*}(0)=\delta_{T}(\theta, 0)+T \mathrm{E} \varepsilon^{+}(0) \quad \text { a.s. }
\end{gathered}
$$

If $\gamma \in(0,1)$, then condition $\mathbf{C}$ implies that

$$
\begin{aligned}
\mathrm{P}\left(\left\|\bar{u}_{T}\right\| \geq r\right) & \leq \mathrm{P}\left(\left\{\left\|\bar{u}_{T}\right\| \geq r\right\} \cap\left\{Q_{T}^{*}\left(\bar{u}_{T}\right) \leq \delta_{T}(\theta, 0)+T \mathrm{E} \varepsilon^{+}(0)\right\}\right) \\
\leq & \mathrm{P}\left(\inf _{u \in \tilde{U}_{T}^{c}(\theta) \backslash V(r)} T^{-1} Q_{T}^{*}(u) \leq T^{-1} \delta_{T}(\theta, 0)+\mathrm{E} \varepsilon^{+}(0)\right) \\
\leq & \mathrm{P}\left(\inf _{u \in \tilde{U}_{T}^{c}(\theta) \backslash V(r)} T^{-1} Q_{T}^{*}(u)\right. \\
\leq & \left.\leq T^{-1} \delta_{T}(\theta, 0)+\inf _{u \in \tilde{U}_{T}^{c}(\theta) \backslash V(r)} T^{-1} \mathrm{E} Q_{T}^{*}(u)-\Delta(r)\right) \\
\leq & \mathrm{P}\left(T^{-1} \delta_{T}(\theta, 0) \geq(1-\gamma) \Delta(r)\right) \\
& +\mathrm{P}\left(\inf _{u \in \tilde{U}_{T}^{c}(\theta) \backslash V(r)} T^{-1} Q_{T}^{*}(u)-\inf _{u \in \tilde{U}_{T}^{c}(\theta) \backslash V(r)} T^{-1} \mathrm{E} Q_{T}^{*}(u) \leq-\gamma \Delta(r)\right) \\
= & \mathrm{P}_{1}+\mathrm{P}_{2} .
\end{aligned}
$$

By Chebyshev's inequality,

$$
\begin{gathered}
\mathrm{P}_{1} \leq \frac{T^{-2} \mathrm{E} \delta_{T}^{2}(\theta, 0)}{((1-\gamma) \Delta(r))^{2}}, \\
T^{-2} \mathrm{E} \delta_{T}^{2}(\theta, 0)=T^{-2} \mathrm{E}\left(\int_{0}^{T} \rho_{\beta}(\varepsilon(t)) d t-T \mathrm{E} \varepsilon^{+}(0)\right)^{2} \\
=T^{-2} \int_{0}^{T} \int_{0}^{T} \mathrm{E} \rho_{\beta}(\varepsilon(t)) \rho_{\beta}(\varepsilon(s)) d t d s-\left(\mathrm{E} \varepsilon^{+}(0)\right)^{2} .
\end{gathered}
$$

Property III of the function $\rho_{\beta}$ implies that

$$
\mathrm{E} \rho_{\beta}^{2}(\varepsilon(t)) \leq \bar{\beta}^{2} \mathrm{E} \varepsilon^{2}(0)=K<\infty .
$$


This means that the decomposition

$$
\rho_{\beta}(G(u))=\sum_{m=0}^{\infty} \frac{c_{m}}{m !} H_{m}(u), \quad c_{m}=\int_{\mathbb{R}} \rho_{\beta}(G(u)) H_{m}(u) \varphi(u) d u, \quad m \geq 0,
$$

is well defined in the Hilbert space $L_{2}(\mathbb{R}, \varphi(u) d u)$, where

$$
H_{m}(u)=(-1)^{m} e^{u^{2} / 2} \frac{d^{m}}{d u^{m}} e^{-u^{2} / 2}, \quad m \geq 0,
$$

are the Chebyshev-Hermite polynomials and where $\varphi(u)=(2 \pi)^{-1 / 2} e^{-u^{2} / 2}$ is the standard Gaussian density. Note that

$$
\mathrm{E} H_{m}(\xi(t)) H_{k}(\xi(s))=\delta_{m}^{k} m ! B^{m}(t-s) .
$$

Hence

$$
\begin{gathered}
\rho_{\beta}(G(\xi(t)))=\sum_{m=0}^{\infty} \frac{c_{m}}{m !} H_{m}(\xi(t)), \\
\mathrm{E} \rho_{\beta}(\varepsilon(t)) \rho_{\beta}(\varepsilon(s))=\sum_{m=0}^{\infty} \frac{c_{m}^{2}}{m !} B^{m}(t-s) .
\end{gathered}
$$

In particular,

$$
\mathrm{E} \rho_{\beta}^{2}(\varepsilon(0))=\sum_{m=0}^{\infty} \frac{c_{m}^{2}}{m !} \leq K<\infty .
$$

Since $\mathrm{E} \rho_{\beta}(\varepsilon(0))=\mathrm{E} \varepsilon^{+}(0)=c_{0}$, we have

$$
\begin{aligned}
T^{-2} \mathrm{E} \delta_{T}^{2}(\theta, 0) & =T^{-2} \int_{0}^{T} \int_{0}^{T}\left(\left(\sum_{m=0}^{\infty} \frac{c_{m}^{2}}{m !} B^{m}(t-s)\right)-\left(\mathrm{E} \varepsilon^{+}(0)\right)^{2}\right) d t d s \\
& =T^{-2} \int_{0}^{T} \int_{0}^{T} \sum_{m=1}^{\infty} \frac{c_{m}^{2}}{m !} B^{m}(t-s) d t d s \leq K T^{-2} \int_{0}^{T} \int_{0}^{T} B(t-s) d t d s .
\end{aligned}
$$

We need the following result of $[9]$ to estimate the latter integral.

Lemma 1. Let $\eta \geq 0$ be a number and let $f(t, s)$ be a measurable function defined on $(0, \infty) \times(0, \infty)$. Assume that the integral

$$
\int_{0}^{\beta} \int_{0}^{\beta} \frac{f(t, s)}{|t-s|^{\eta}} d t d s
$$

converges for some $0<\beta<\infty$. Let a slowly varying function $L$ be bounded on every bounded interval of $\mathbb{R}_{+}$. If $\eta>0$, then

$$
\int_{0}^{\beta} \int_{0}^{\beta} f(t, s) \frac{L(T|t-s|)}{L(T)} d t d s \underset{T \rightarrow \infty}{\longrightarrow} \int_{0}^{\beta} \int_{0}^{\beta} f(t, s) d t d s .
$$

If $\eta=0$, then the same conclusion holds under the additional assumption that the function $L$ is nondecreasing on the axis $(0, \infty)$.

Lemma 1 with $f(t, s)=|t-s|^{-\alpha}$ and $\eta \geq 0$ such that $\alpha+\eta<1$ implies that

$$
\begin{aligned}
\int_{0}^{T} \int_{0}^{T} B(t-s) d t d s & =T^{2} \int_{0}^{1} \int_{0}^{1} B(T(t-s)) d t d s=T^{2} \int_{0}^{1} \int_{0}^{1} \frac{L(T|t-s|)}{T^{\alpha}|t-s|^{\alpha}} d t d s \\
& \sim\left(\int_{0}^{1} \int_{0}^{1} \frac{1}{|t-s|^{\alpha}} d t d s\right) \cdot \frac{L(T)}{T^{\alpha-2}}=\frac{2}{(1-\alpha)(2-\alpha)} \cdot \frac{L(T)}{T^{\alpha-2}}
\end{aligned}
$$


as $T \rightarrow \infty$, where the relation $a(T) \sim b(T)$ as $T \rightarrow \infty$ means that

$$
\lim _{T \rightarrow \infty} \frac{a(T)}{b(T)}=1 .
$$

Therefore

$$
\begin{aligned}
\mathrm{P}_{1} & \leq \frac{K T^{-2} \int_{0}^{T} \int_{0}^{T} B(t-s) d t d s}{((1-\gamma) \Delta(r))^{2}} \\
& \sim \frac{2 K}{(1-\alpha)(2-\alpha)((1-\gamma) \Delta(r))^{2}} \cdot \frac{L(T)}{T^{\alpha}}=O(B(T))
\end{aligned}
$$

as $T \rightarrow \infty$.

On the other hand,

$$
\begin{gathered}
\mathrm{P}_{2} \leq \mathrm{P}\left(\inf _{u \in \tilde{U}_{T}^{c}(\theta) \backslash V(r)} T^{-1} \delta_{T}(\theta, u) \leq-\gamma \Delta(r)\right) \\
Q_{T}^{*}(u)=\int_{0}^{T} \rho_{\beta}(X(t)-h(t, u)) d t \leq \int_{0}^{T} \rho_{\beta}(\varepsilon(t)) d t+\int_{0}^{T} \rho_{\beta}(h(t, 0)-h(t, u)) d t \\
=\int_{0}^{T} \rho_{\beta}(\varepsilon(t)) d t+\Psi_{T}(0, u)
\end{gathered}
$$

in view of property IV. Using bound (3) we prove the inequality

$$
\Psi_{T}(0, u)-\int_{0}^{T} \rho_{\beta}(-\varepsilon(t)) d t \leq Q_{T}^{*}(u) \leq \Psi_{T}(0, u)+\int_{0}^{T} \rho_{\beta}(\varepsilon(t)) d t .
$$

Further

$$
\begin{aligned}
T^{-1} \delta_{T}(\theta, u)= & T^{-1}\left(Q_{T}^{*}(u)-\mathrm{E} Q_{T}^{*}(u)\right) \\
\geq & T^{-1}\left(\Psi_{T}(0, u)-\int_{0}^{T} \rho_{\beta}(-\varepsilon(t)) d t-\mathrm{E} Q_{T}^{*}(u)\right) \\
= & T^{-1}\left(-\int_{0}^{T} \rho_{\beta}(-\varepsilon(t)) d t+\Psi_{T}(0, u)\right) \\
& -T^{-1}\left(\mathrm{E} \int_{0}^{T} \rho_{\beta}(X(t)-h(t, u)) d t\right) \\
= & T^{-1}\left(-\int_{0}^{T} \rho_{\beta}(-\varepsilon(t)) d t-T \mathrm{E} \varepsilon^{+}(0)\right) .
\end{aligned}
$$

Hence

$$
T^{-1} \delta_{T}(\theta, u) \geq-T^{-1} \int_{0}^{T} \rho_{\beta}(-\varepsilon(t)) d t-\mathrm{E} \varepsilon^{+}(0),
$$

whence

$$
\mathrm{P}_{2} \leq \mathrm{P}\left(T^{-1} \int_{0}^{T} \rho_{\beta}(-\varepsilon(t)) d t+\mathrm{E} \varepsilon^{+}(0) \geq \gamma \Delta(r)\right)
$$


Let $r=r_{0}$ and $\gamma=2 / a_{0}$, where the numbers $r_{0}$ and $a_{0}$ are defined in condition $\mathbf{C}$. Then (10) together with Chebyshev's inequality implies that

$$
\begin{aligned}
\mathrm{P}_{2} & \leq \mathrm{P}\left(T^{-1} \int_{0}^{T} \rho_{\beta}(-\varepsilon(t)) d t+\mathrm{E} \varepsilon^{+}(0) \geq \gamma \Delta(r)\right) \\
& =\mathrm{P}\left(T^{-1} \int_{0}^{T} \rho_{\beta}(-\varepsilon(t)) d t+\mathrm{E} \varepsilon^{+}(0) \geq \frac{2}{a_{0}} \Delta\left(r_{0}\right)\right) \\
& =\mathrm{P}\left(T^{-1} \int_{0}^{T} \rho_{\beta}(-\varepsilon(t)) d t+\mathrm{E} \varepsilon^{+}(0) \geq \frac{2}{a_{0}}\left(a_{0} \mathrm{E} \varepsilon^{+}(0)+\Delta_{0}\right)\right) \\
& =\mathrm{P}\left(T^{-1} \int_{0}^{T}\left(\rho_{\beta}(-\varepsilon(t))-\mathrm{E} \varepsilon^{+}(0)\right) d t \geq \frac{2 \Delta_{0}}{a_{0}}\right) \\
& \leq\left(\frac{a_{0}}{2 \Delta_{0}}\right)^{2} T^{-2} \mathrm{E}\left(\int_{0}^{T}\left(\rho_{\beta}(-\varepsilon(t))-\mathrm{E} \varepsilon^{+}(0)\right) d t\right)^{2} .
\end{aligned}
$$

Following the method used to estimate the probability $\mathrm{P}_{1}$, we consider the representation in the Hilbert space $L_{2}(\mathbb{R}, \varphi(u) d u)$ :

$$
\rho_{\beta}(-G(u))=\sum_{m=0}^{\infty} \frac{d_{m}}{m !} H_{m}(u),
$$

where

$$
d_{m}=\int_{\mathbb{R}} \rho_{\beta}(-G(u)) H_{m}(u) \varphi(u) d u, \quad m \geq 0,
$$

and

$$
\begin{gathered}
\mathrm{E} \rho_{\beta}(-\varepsilon(t)) \rho_{\beta}(-\varepsilon(s))=\sum_{m=0}^{\infty} \frac{d_{m}^{2}}{m !} B^{m}(t-s), \\
\mathrm{E} \rho_{\beta}^{2}(-\varepsilon(0))=\sum_{m=0}^{\infty} \frac{d_{m}^{2}}{m !} \leq K<\infty .
\end{gathered}
$$

Since $c_{0}=d_{0}$ by condition (4), we get

$$
T^{-2} \mathrm{E}\left(\int_{0}^{T}\left(\rho_{\beta}(-\varepsilon(t))-\mathrm{E} \varepsilon^{+}(0)\right) d t\right)^{2} \leq K T^{-2} \int_{0}^{T} \int_{0}^{T} B(t-s) d t d s .
$$

As above, the right-hand side of (11) is $O(B(T))$ as $T \rightarrow \infty$.

It remains to estimate the probability

$$
\begin{aligned}
\mathrm{P}\left(r_{0}>\left\|\bar{u}_{T}\right\| \geq r\right) \leq & \mathrm{P}\left(T^{-1} \delta_{T}(\theta, 0) \geq\left(1-\gamma^{\prime}\right) \Delta(r)\right) \\
& +\mathrm{P}\left(\inf _{u \in\left(V^{c}\left(r_{0}\right) \backslash V(r)\right) \cap \tilde{U}_{T}^{c}(\theta)} T^{-1} \delta_{T}(\theta, u) \leq-\gamma^{\prime} \Delta(r)\right) \\
\leq & \mathrm{P}\left(\sup _{V^{c}\left(r_{0}\right) \cap \tilde{U}_{T}^{c}(\theta)} T^{-1}\left|\delta_{T}(\theta, u)\right| \geq \gamma^{\prime} \Delta(r)\right)+O(B(T))
\end{aligned}
$$

for $\gamma^{\prime} \in(0,1)$.

Let $F^{(1)}, \ldots, F^{(l)} \subset V^{c}\left(r_{0}\right)=\left\{u \in \mathbb{R}^{q}:\|u\| \leq r_{0}\right\}$ be closed sets, whose diameters do not exceed $\delta$ defined by (15) with $r=r_{0}$. Let $\varepsilon=\nu \Delta(r) \gamma^{\prime} /(2 \bar{\beta})$, where $\nu \in(0,1)$ is a 
certain number, and let

$$
\bigcup_{i=1}^{l} F^{(i)}=V^{c}\left(r_{0}\right)
$$

Fix $u_{i} \in F^{(i)} \cap \tilde{U}_{T}^{c}(\theta), i=1, \ldots, l$. Then

$$
\begin{aligned}
\mathrm{P}_{3} & =\mathrm{P}\left(\sup _{u \in \bigcup_{i=1}^{l}\left(F^{(i)} \cap \tilde{U}_{T}^{c}(\theta)\right)} T^{-1}\left|\delta_{T}(\theta, u)\right| \geq \gamma^{\prime} \Delta(r)\right) \\
& =\mathrm{P}\left(\bigcup_{i=1}^{l}\left(\sup _{u \in\left(F^{(i)} \cap \tilde{U}_{T}^{c}(\theta)\right)} T^{-1}\left|\delta_{T}(\theta, u)\right| \geq \gamma^{\prime} \Delta(r)\right)\right) \\
& \leq \sum_{i=1}^{l} \mathrm{P}\left(\sup _{u^{\prime}, u^{\prime \prime} \in\left(F^{(i)} \cap \tilde{U}_{T}^{c}(\theta)\right)} T^{-1}\left|\delta_{T}\left(\theta, u^{\prime}\right)-\delta_{T}\left(\theta, u^{\prime \prime}\right)\right|+T^{-1}\left|\delta_{T}\left(\theta, u_{i}\right)\right| \geq \gamma^{\prime} \Delta(r)\right) .
\end{aligned}
$$

According to property $\mathbf{V}$ we have

$$
\begin{aligned}
\left|\delta_{T}\left(\theta, u^{\prime}\right)-\delta_{T}\left(\theta, u^{\prime \prime}\right)\right| \leq & \left|Q_{T}^{*}\left(u^{\prime}\right)-Q_{T}^{*}\left(u^{\prime \prime}\right)\right|+\mathrm{E}\left|Q_{T}^{*}\left(u^{\prime}\right)-Q_{T}^{*}\left(u^{\prime \prime}\right)\right| \\
\leq & \int_{0}^{T}\left|\rho_{\beta}\left(X(t)-h\left(t, u^{\prime}\right)\right)-\rho_{\beta}\left(X(t)-h\left(t, u^{\prime \prime}\right)\right)\right| d t \\
& +\mathrm{E} \int_{0}^{T}\left|\rho_{\beta}\left(X(t)-h\left(t, u^{\prime}\right)\right)-\rho_{\beta}\left(X(t)-h\left(t, u^{\prime \prime}\right)\right)\right| d t \\
\leq & 2 \bar{\beta} \Phi_{1 T}\left(u^{\prime}, u^{\prime \prime}\right) .
\end{aligned}
$$

Considering condition (5), we conclude that

$$
\sup _{u^{\prime}, u^{\prime \prime} \in\left(F^{(i)} \cap \tilde{U}_{T}^{c}(\theta)\right)} 2 T^{-1} \bar{\beta} \Phi_{1 T}\left(u^{\prime}, u^{\prime \prime}\right) \leq 2 \bar{\beta} \varepsilon=\nu \gamma^{\prime} \Delta(r) .
$$

Thus

$$
\mathrm{P}_{3} \leq \sum_{i=1}^{l} \mathrm{P}\left(T^{-1}\left|\delta_{T}\left(\theta, u_{i}\right)\right| \geq(1-\nu) \gamma^{\prime} \Delta(r)\right) .
$$

Every term of the latter sum is estimated separately:

where

$$
\mathrm{P}\left(T^{-1}\left|\delta_{T}\left(\theta, u_{i}\right)\right| \geq(1-\nu) \gamma^{\prime} \Delta(r)\right) \leq \frac{T^{-2} \mathrm{E} \delta_{T}^{2}\left(\theta, u_{i}\right)}{\left((1-\nu) \gamma^{\prime} \Delta(r)\right)^{2}},
$$

$$
\begin{aligned}
\delta_{T}\left(\theta, u_{i}\right) & =Q_{T}^{*}\left(u_{i}\right)-\mathrm{E} Q_{T}^{*}\left(u_{i}\right) \\
& =\int_{0}^{T} \rho_{\beta}\left(X(t)-h\left(t, u_{i}\right)\right) d t-\mathrm{E} \int_{0}^{T} \rho_{\beta}\left(X(t)-h\left(t, u_{i}\right)\right) d t .
\end{aligned}
$$

Put $\Delta h\left(t, u_{i}\right)=h\left(t, u_{i}\right)-h(t, 0)$. Then $\rho_{\beta}\left(X(t)-h\left(t, u_{i}\right)\right)=\rho_{\beta}\left(\varepsilon(t)-\Delta h\left(t, u_{i}\right)\right)$ and

$$
\begin{aligned}
\mathrm{E} \delta_{T}^{2}\left(\theta, u_{i}\right)= & \int_{0}^{T} \int_{0}^{T} \mathrm{E} \rho_{\beta}\left(\varepsilon(t)-\Delta h\left(t, u_{i}\right)\right) \rho_{\beta}\left(\varepsilon(s)-\Delta h\left(s, u_{i}\right)\right) d t d s \\
& -\left(\int_{0}^{T} \mathrm{E} \rho_{\beta}\left(\varepsilon(t)-\Delta h\left(t, u_{i}\right)\right) d t\right)^{2} .
\end{aligned}
$$

Let

$$
\rho_{\beta}\left(\varepsilon(t)-\Delta h\left(t, u_{i}\right)\right)=Z(\varepsilon(t))=Z(G(\xi(t))) .
$$


Since, for every fixed $t \in[0, T]$,

$$
\begin{aligned}
\mathrm{E} Z^{2}(\varepsilon(0)) & =\mathrm{E} \rho_{\beta}^{2}\left(\varepsilon(t)-\Delta h\left(t, u_{i}\right)\right) \leq \bar{\beta}^{2} \mathrm{E}\left(\varepsilon(0)-\Delta h\left(t, u_{i}\right)\right)^{2} \\
& =\bar{\beta}^{2}\left(\mathrm{E} \varepsilon^{2}(0)+\Delta h^{2}\left(t, u_{i}\right)\right)<\infty,
\end{aligned}
$$

the function $Z(G(\cdot))$ can be decomposed in the Hilbert space $L_{2}(\mathbb{R}, \varphi(x) d x)$ with respect to the Chebyshev-Hermite polynomials, namely

$$
\begin{gathered}
Z(G(x))=\rho_{\beta}\left(G(x)-\Delta h\left(t, u_{i}\right)\right)=\sum_{m=0}^{\infty} \frac{C_{m}\left(t, u_{i}\right)}{m !} H_{m}(x), \\
C_{m}\left(t, u_{i}\right)=\int_{\mathbb{R}} \rho_{\beta}\left(G(x)-\Delta h\left(t, u_{i}\right)\right) H_{m}(x) \varphi(x) d x, \quad m \geq 0, \\
C_{0}\left(t, u_{i}\right)=\mathrm{E} Z(\varepsilon(0)) .
\end{gathered}
$$

Further

$$
\mathrm{E} Z(G(\xi(t))) Z(G(\xi(s)))=\sum_{m=0}^{\infty} \frac{C_{m}\left(t, u_{i}\right) C_{m}\left(s, u_{i}\right)}{m !} B^{m}(t-s) .
$$

Then

$$
\begin{aligned}
T^{-2} \mathrm{E} & \delta_{T}^{2}\left(\theta, u_{i}\right) \\
& =T^{-2} \int_{0}^{T} \int_{0}^{T}\left(\sum_{m=0}^{\infty} \frac{C_{m}\left(t, u_{i}\right) C_{m}\left(s, u_{i}\right)}{m !} B^{m}(t-s)-C_{0}\left(t, u_{i}\right) C_{0}\left(s, u_{i}\right)\right) d t d s \\
& =T^{-2} \int_{0}^{T} \int_{0}^{T} \sum_{m=1}^{\infty} \frac{C_{m}\left(t, u_{i}\right) C_{m}\left(s, u_{i}\right)}{m !} B^{m}(t-s) d t d s \\
& \leq T^{-2} \int_{0}^{T} \int_{0}^{T} \sum_{m=1}^{\infty} \frac{C_{m}^{2}\left(t, u_{i}\right)}{m !} B^{m}(t-s) d t d s \\
& \leq T^{-2} \int_{0}^{T} \int_{0}^{T}\left(\sum_{m=1}^{\infty} \frac{C_{m}^{2}\left(t, u_{i}\right)}{m !}\right) B(t-s) d t d s \\
& \leq T^{-2} \int_{0}^{T} \int_{0}^{T} \mathrm{E} Z^{2}(\varepsilon(0)) B(t-s) d t d s \\
& \leq T^{-2} \int_{0}^{T} \int_{0}^{T} \bar{\beta}^{2}\left(\mathrm{E}\left(\varepsilon^{2}(0)+\Delta h^{2}\left(t, u_{i}\right)\right) B(t-s) d t d s\right. \\
& \leq \bar{\beta}^{2} T^{-2} \int_{0}^{T} \int_{0}^{T} \Delta h^{2}\left(t, u_{i}\right) B(t-s) d t d s+O(B(T))
\end{aligned}
$$

as $T \rightarrow \infty$. Since

$$
T^{-1} \int_{0}^{T} B(s) d s=O(B(T))
$$

(see [7, Chapter 2, Theorem 2.7]), we estimate the latter integral as follows:

$$
\begin{aligned}
T^{-2} \int_{0}^{T} \int_{0}^{T} \Delta h^{2}\left(t, u_{i}\right) B(t-s) d t d s & =T^{-1} \int_{0}^{T} \Delta h^{2}\left(t, u_{i}\right)\left(T^{-1} \int_{0}^{T} B(t-s) d s\right) d t \\
& \leq T^{-1} \Phi_{2 T}\left(u_{i}, 0\right) T^{-1} \int_{-T}^{T} B(s) d s \\
& \leq 2 \sigma(r) T^{-1} \int_{0}^{T} B(s) d s=O(B(T)) .
\end{aligned}
$$


This relation implies that

$$
T^{-2} \mathrm{E} \delta_{T}^{2}\left(\theta, u_{i}\right)=O(B(T))
$$

as $T \rightarrow \infty$. Thus

$$
\mathrm{P}\left(T^{-1}\left|\delta_{T}\left(\theta, u_{i}\right)\right| \geq(1-\nu) \gamma^{\prime} \Delta(r)\right)=O(B(T)), \quad i=1, \ldots, l .
$$

Now we obtain from (12) and (13) that

$$
\mathrm{P}_{3}=O(B(T)) .
$$

\section{Concluding Remarks}

Sufficient conditions are found in the paper for weak consistency of the KoenkerBassett estimator in the quantile regression model with long-range memory noise and continuous time. The proof of the asymptotic normality of the estimator will be published elsewhere.

\section{BIBLIOGRAPHY}

1. J. Beran, Statistics for Long-Memory Processes, Chapman and Hall, New York, 1994. MR 1304490 (96b:62138)

2. N. N. Leonenko, Limit Theorems for Random Fields with Singular Spectrum, Kluwer Academic Publishers, Dordrecht, 1999. MR1687092 (2000k:60102)

3. P. Doukhan, G. Oppenheim, and M. S. Taqqu, Theory and Applications of Long-Range Dependence, Birkhäuser, Boston, 2003. MR1956041 (2003h:60004)

4. G. Bassett and R. Koenker, Regression quantile, Econometrica (G. Bassett and R. Koenker, eds.), vol. 46, 1978, pp. 33-50. MR0474644 (57:14279)

5. I. V. Orlovs'kiü, Consistence of Koenker-Bassett estimators in nonlinear regression models, Naukovi visti NTUU "KPI" 35 (2004), no. 3, 144-150. (Ukrainian)

6. O. V. Ivanov and I. V. Orlovs'kiū, Asymptotic normality of Koenker-Bassett estimators in nonlinear regression models, Teor. Imovir. Mat. Stat. 72 (2005), 30-41; English transl. in Theory Probab. Math. Statist. 72 (2006), 33-45. MR.2168134 (2007b:62029)

7. E. Seneta, Regularly Varying Functions, Springer-Verlag, Berlin-New York, 1976. MR0453936 $(56: 12189)$

8. J. Pfanzagl, On the measurability and consistency of minimum contrast estimates, Metrika $\mathbf{1 4}$ (1969), 249-272.

9. A. V. Ivanov and I. V. Orlovsky, $L_{p}$ estimates in nonlinear regression with long-range dependence, Theory Stoch. Process. 7(23) (2002), no. 3-4, 38-49.

Department of Mathematical Analysis and Probability Theory, National Technical University of Ukraine "Kyiv Polytechnic Institute", Peremogy Avenue 37, Kyiv-56 03056, UkraINE

E-mail address: sim_ka@i.ua

Received 1/DEC/2009

Translated by OLEG KLESOV 